https://doi.org/10.15407/ujpe65.3.217

A.G. MAGNER, ${ }^{1}$ M.I. GORENSTEIN,${ }^{2}$ U.V. GRYGORIEV ${ }^{1}$

${ }^{1}$ Institute for Nuclear Research, Nat. Acad. of Sci. of Ukraine (47, Prosp. Nauky, Kyiv 03028, Ukraine; e-mail: magner@kinr.kiev.ua)

${ }^{2}$ Bogolyubov Institute for Theoretical Physics, Nat. Acad. of Sci. of Ukraine (14b, Metrologichna Str., Kyiv 03143, Ukraine)

\title{
VELOCITY AND ABSORPTION COEFFICIENT OF SOUND WAVES IN CLASSICAL GASES
}

\begin{abstract}
The velocity and absorption coefficient of plane sound waves in classical gases are obtained by solving the Boltzmann kinetic equation. This is done within the linear response theory as a reaction of the single-particle distribution function to a periodic external field. The nonperturbative dispersion equation is derived in the relaxation time approximation and solved numerically. The obtained theoretical results demonstrate an universal dependence of the sound velocity and scaled absorption coefficient on the variable $\omega \tau$, where $\omega$ is the sound frequency, and $\tau^{-1}$ is the particle collision frequency. In the region of $\omega \tau \sim 1$, a transition from the frequent- to rare-collision regime takes place. The sound velocity increases sharply, and the scaled absorption coefficient has a maximum - both theoretical findings are in agreement with the data.

Keywords: hydrodynamics, kinetic approach, ultrasonic plane sound waves, velocity, absorption.
\end{abstract}

\section{Introduction: Hydrodynamics and Kinetics}

Sound waves in classical gases were studied intensively within the hydrodynamical approach (see, e.g., Ref. [1]). The sound velocity $c_{0}$ in this approach is equal to

$c_{0}=\left(\frac{c_{p} k_{\mathrm{B}} T}{c_{v} m}\right)^{1 / 2}$,

where $k_{\mathrm{B}}$ is the Boltzmann constant, $T$ is the system temperature, $m$ the particle mass, $c_{p}$ and $c_{v}$ are the specific heat capacity at a constant pressure and a constant volume, respectively. The sound velocity (1) appears to be independent of the sound wave frequency $\omega$ and approximately equals the thermal particle velocity. For absorbed plane sound waves (APSW), the wave amplitude decreases as $\exp (-\gamma z)$ after propagating the distance $z$. The absorption coefficient $\gamma$ is usually evaluated from the Stokes relation [1],

$\gamma=\frac{\omega^{2}}{2 m n c_{0}^{3}}\left[\frac{4}{3} \eta+\zeta+\left(\frac{1}{c_{v}}-\frac{1}{c_{p}}\right) \kappa\right]$,

(C) A.G. MAGNER, M.I. GORENSTEIN, U.V. GRYGORIEV, 2020

ISSN 2071-0194. Ukr. J. Phys. 2020. Vol. 65, No. 3 where $n$ is the particle number density, $\eta$ the shear viscosity, $\zeta$ the bulk viscosity, and $\kappa$ the thermal conductivity.

Within the hydrodynamic approach, the kinetic coefficients are phenomenological constants. For calculations of the kinetic coefficients in Eq. (2), one needs the kinetic theory. The global equilibrium of a classical gas is described then by the Maxwell distribution function of particle's momentum $\mathbf{p}$ with $p \equiv|\mathbf{p}|$ :

$f_{\mathrm{GE}}(\mathbf{p})=\frac{n}{\left(2 \pi m k_{\mathrm{B}} T\right)^{3 / 2}} \exp \left(-\frac{p^{2}}{2 m k_{\mathrm{B}} T}\right)$.

In this relation, the particle number density $n$ and temperature $T$ are independent of the spacial coordinates $\mathbf{r}$ and time $t$. The equilibrium in a classical gas is achieved by successive two-body collisions with the elastic cross-section $\sigma$ equal to $\pi d^{2}$ for hard-sphere particles with diameter $d$.

The average value of the thermal velocity $v$ can be calculated from Eq. (3) as $(\mathbf{p} \equiv m \mathbf{v}$ and $v \equiv|\mathbf{v}|)$ :

$\bar{v}=\frac{\int p^{2} d p(p / m) f_{\mathrm{GE}}(p)}{\int p^{2} d p f_{\mathrm{GE}}(p)}=\left(\frac{8 k_{\mathrm{B}} T}{\pi m}\right)^{1 / 2}$. 

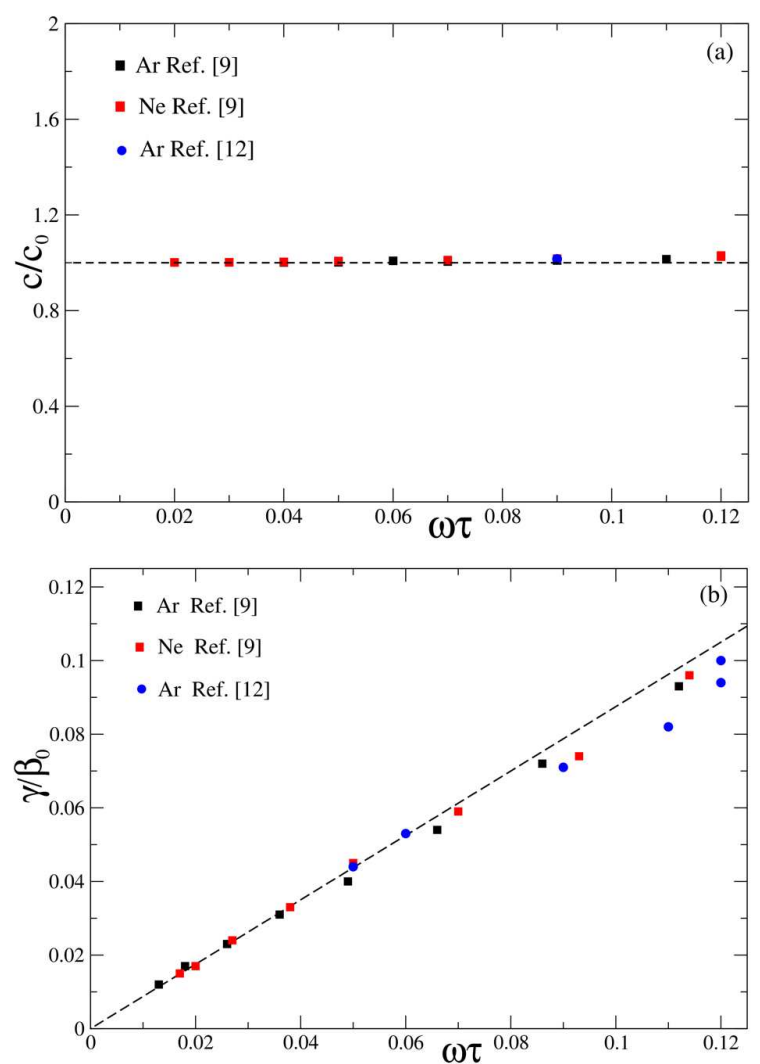

Fig. 1. The speed of sound $c / c_{0}(a)$ and the scaled absorption coefficient $\gamma / \beta_{0}(b)$ as functions of $\omega \tau$ at low frequencies. The experimental data are taken from Refs. [9, 12]. Dashed lines present Eq. (1) in (a) and the Stokes formula (9) in (b)

The particle mean-free path can be found analytically as a function of $\sigma$ and $n[2,3]$ :

$l=(\sqrt{2} \sigma n)^{-1}$.

From Eqs. (4) and (5), one finds the collision frequency as

$\tau^{-1} \equiv \bar{v} / l=4 \sigma n \sqrt{\frac{k_{\mathrm{B}} T}{\pi m}}$.

In terms of the above quantities, the shear viscosity $\eta$ can be calculated as

$\eta=\frac{5 \pi}{32} n m l \bar{v}=\frac{5 \sqrt{\pi}}{16} \frac{\sqrt{m k_{\mathrm{B}} T}}{\sigma}$.

The numerical coefficient in Eq. (7) was found by Chapman and Enskog (see, e.g., Ref. [4]). The thermal conductivity can be then found as

$\kappa=\frac{15}{4} \eta$, and the bulk viscosity for non-relativistic monoatomic gases equals zero, $\zeta=0$ (see, e.g., [5]).

The molecular kinetic scheme based on the above equations is self-consistent for dilute gases, when the mean free path $l$ is much larger than the size of particles, $l \gg d$. In this case, the excluded volume effects due to the particle hard-core repulsion appear to be negligible, i.e., the gas pressure, $P=n k_{\mathrm{B}} T$, and specific heat capacity, $c_{v}=3 / 2$ and $c_{p}=5 / 2$, are equal to their ideal gas values with high accuracy [6]. In what follows, the thermodynamical relations (4)-(6) are assumed to be valid, i.e., our consideration is restricted to the case of dilute classical gases. Equations (7) and (8) are the leading terms of the perturbation expansion in the small Knudsen parameter, $\mathcal{K} \equiv \omega \tau \ll 1$. This corresponds to the so-called frequent collision regime (FCR).

Using the above equations, one finds the FCR scaled absorption coefficient from the Stokes formula (2):

$\frac{\gamma}{\beta_{0}}=\frac{7}{8} \omega \tau$

where $\beta_{0} \equiv \omega / c_{0}$ is the wave number. As shown in Fig. 1, both $c_{0}$ and $\gamma / \beta_{0}$ given by Eqs. (1) and (9), respectively, are supported by the data at small $\omega \tau$.

In most practical cases, the inequality $l \ll \lambda$, where $\lambda \cong 2 \pi c_{0} / \omega$ is the sound wavelength, is satisfied, and, thus, the FCR is valid. For example, for gases under the normal conditions, one gets $\omega \tau \sim 10^{-8}$ $10^{-5}$ for the audible frequency region. Calculating $\gamma$ from Eq. (9), one finds $\gamma^{-1} \sim l(\omega \tau)^{-2} \sim 10^{5}-10^{8} \mathrm{~cm}$ $\left(c_{0} \sim \bar{v}\right)$, i.e., the audible APSW propagate $1-10^{3}$ kilometers before its amplitude decreases by the factor of $e^{-1}$. The absorption of these waves is indeed rather weak. Note also that all kinetic coefficients in the FCR depend only on the equilibrium gas quantities. For example, the shear viscosity (7) and thermal conductivity (8) are independent of the sound frequency.

Equations (1) and (9) are, however, in a contradiction with the existing data at $\omega \tau \gtrsim 1$; see critical comments, e.g., in Refs. $[3,7,8]$. This is a transition region from the FCR to the rare-collision regime (RCR). The RCR corresponds to large values of the Knudsen parameter, $\mathcal{K} \equiv \omega \tau \gg 1$. The conditions of the RCR emerge at a small particle number density (or small pressure), where $l$ increases as $n^{-1}$ by Eq. (5), and for large sound-wave frequencies $\omega$. The basic experiments for sound waves in classical 
gases at large values of $\omega \tau$ (the so-called ultrasonic waves) were done by Greenspan [9-11] and MeyerSessler [12]. Many works were devoted to a comparison of the theoretical and experimental results for the sound velocity and absorption. One of the directions on the early study of this problem was related to the accounting for high p-moments of the Boltzmann kinetic equation (BKE) within the method of moments, called the Bartnett [13] and super-Bartnett [14] models to obtain solutions for $\omega \tau<1$, see also Refs. [7, 8, 15-17]. An alternative kinetic approach is based on the well-known Bhatnagar-Gross-Krook model [18] and its generalization by Gross and Jackson [19], see also Refs. [3, 8]. The main idea was to use the linearized BKE with the relaxation $\tau$ approximation for the integral collision term and to express exactly the solution of this equation in terms of local dynamical variations of the particle number density, $\delta n(\mathbf{r}, t)$, the velocity field $\mathbf{u}$ and temperature variations $\delta T(\mathbf{r}, t)$. Using the conditions to restore the conservation relationships (violated, generally speaking, by the $\tau$ approximation, see also Refs. $[20-22]$ ), one can then approximately obtain the dispersion equation for the sound velocity [21]. They suggested also to apply the boundary conditions on the walls of a conductor pipe for the particle gas motion. Such solutions were found [3, 8, 23-29] for the simplest case of the diffused boundary conditions on a plane surface of the semiinfinite gas system. Usually, these boundary conditions for the distribution function are considered by assuming the mirror or diffuse reflections of particles from the boundary [3032]. The linearization of the BKE for calculations of the sound velocity and absorption in the solutions in terms of a plane wave can be grounded in the case of a small influence of boundary conditions of the sound propagation on the size of a conductor pipe much larger than the wavelength; see, e.g., Ref. [33] for such numerical BKE solutions for the sound velocity and absorption without using the boundary conditions. As shown in Ref. [34] for the kinetic equation with mirror boundary conditions at the plane surfaces of a slab and in Ref. [35] for the monopole vibrations with diffuse boundary conditions at the spherical surface, the sound solutions are, in fact, rather different from a plane wave, because they depend very much on the type of the boundary conditions and the choice of the boundary geometry itself.
Both the FCR and RCR for the sound velocity and absorption analytically in terms of a simple plane wave without the use of different boundary conditions and, therefore, independent of their specific properties have been studied within the linearized Boltzmann kinetic equation in our recent paper [36]. The approximate expansions for small and large values of $\mathcal{K}$ were obtained analytically by using the $\tau$ approximation for the collision integral with a constant $\tau$, that is independent of the particle velocity. The dispersion equation for the sound velocity and absorption was derived approximately analytically within the linear response theory [37] following the ideas of the BGK model [18]. In the present paper, our theoretical approach is worked out and compared carefully with the available data.

\section{Boltzmann Kinetic Approach}

We consider the BKE for the single-particle distribution function $f(\mathbf{r}, \mathbf{p}, t)$ of the coordinate $\mathbf{r}$, momentum $\mathbf{p}$, and time $t$ (see, e.g., Ref. [5]) with the external potential $V_{\text {ext }}(z, t)$ (more details can be found in Ref. [37]),

$V_{\text {ext }}(z, t)=\exp (-i \omega t) \int_{-\infty}^{\infty} \frac{\mathrm{d} k}{2 \pi} V_{k} \exp (i k z)$,

where $V_{k}$ is the Fourier amplitudes ${ }^{1}$. The linearized BKE takes the form

$\frac{\partial \delta f}{\partial t}+\frac{p_{z}}{m} \frac{\partial \delta f}{\partial z}-\delta S t[f]=\frac{\partial f_{\mathrm{GE}}}{\partial p_{z}} \frac{\partial V_{\mathrm{ext}}}{\partial z}$,

where $\delta f(\mathbf{r}, \mathbf{p}, t) \equiv f(\mathbf{r}, \mathbf{p}, t)-f_{\mathrm{GE}}(p)$, and the collision term $\delta S t[f]$ is taken in the standard Boltzmann form (see, e.g., Ref. [3]).

A small periodic external field (10) induces the corresponding deviations

$\delta f(z, \mathbf{p}, t)=\exp (-i \omega t) \int_{-\infty}^{\infty} \frac{d k}{2 \pi} f_{k} \exp (i k z)$

with small Fourier amplitudes $f_{k} \propto V_{k}$ (see, e.g., $[22,38,39])$. Equation (11) is assumed to be valid at $|\delta f| / f_{\mathrm{GE}} \ll 1$ in the APSW form with the frequency

1 As usual, the complex number representation is used for convenience, but only the real parts of $f$ and $V_{\text {ext }}$ will be taken as physical quantities. 
$\omega$ propagated along the $z$ axis. For the collision integral $\delta S t$, we use the $\tau$ relaxation-time approximation in the form [37]

$\delta S t[f] \cong-\frac{1}{\tau}\left(\delta f-\delta f_{\mathrm{LE}}\right) \equiv-\frac{1}{\tau} \delta \varphi$,

where $\tau^{-1}$ is a constant given by Eq. (6), and the local equilibrium part of $\delta f$ is related to the wellknown Maxwellian function,

$\delta f_{\mathrm{LE}}=f_{\mathrm{GE}}\left[\frac{\delta n}{n}+p_{z} \frac{\delta u_{z}}{k_{\mathrm{B}} T}+\left(\frac{p^{2}}{2 m k_{\mathrm{B}} T}-\frac{3}{2}\right) \frac{\delta T}{T}\right]$

In Eq. (13), $\delta \varphi \equiv \delta f-\delta f_{\mathrm{LE}}$ appears as the additional component responsible for the sound absorption in a gas through the collision integral $\delta S t[f]$ (13). Note that one has $S t\left[f_{\mathrm{LE}}\right]=0$ at the local equilibrium distribution function $f_{\mathrm{LE}}$ for any parameters $\delta n, \delta T$, and $\delta u_{z}[3,40]$. Thus, just the $\delta \varphi$ term is responsible for all dissipative effects in a gas. In Eq. (14), the variations $\delta n, \delta T$, and $\delta u_{z}$ are small deviations of the particle number density, temperature, and collective velocity, $|\delta n| / n \ll 1,|\delta T| / T \ll 1$, and $\left|\delta u_{z}\right| / \bar{v} \ll 1$, from their GE values $n, T$, and $u_{z}=0$. The conservation of the particle number, momentum, and energy imposes the following requirements $[21,37]$ :

$$
\begin{aligned}
& \int d \mathbf{p} \delta \varphi=0, \quad \int \mathrm{d} \mathbf{p} p_{z} \delta \varphi=0, \\
& \int d \mathbf{p} p^{2} \delta \varphi=0
\end{aligned}
$$

In what follows, for simplicity, we put $\delta T=0$ in Eq. (14), i.e., the effects of thermal conductivity will be neglected. For a constant temperature $T$, only Eq. (15) should be considered. Equation (16) for the energy conservation is then identically satisfied.

The solution of the linearized BKE (11) is found from Eq. (12) by calculating the $k$-integral by the residue method in the following APSW form:

$\delta f(z, \mathbf{p}, t) \propto \exp \left(-i \omega t+i k_{0} z\right)$,

where the poles in the complex $k$ plane are connected with the speed of sound $c$ and absorption coefficient $\gamma$, e.g., as

$k_{0}=\frac{\omega}{c}+i \gamma$
The positions of poles $k_{0}$ can be obtained from the dispersion relation [37]

$$
\begin{aligned}
& \mathrm{D}(w, \mathcal{K}) \equiv\left[\frac{i w}{\xi \mathcal{K}}\left(1+Q_{1}\right)-1\right]\left(3 i \frac{w}{\mathcal{K}} \xi Q_{1}-1\right)+ \\
& +\frac{8}{\pi}\left(\frac{w Q_{1}}{\mathcal{K}}\right)^{2}=0
\end{aligned}
$$

where $w \equiv \omega / k_{0} v_{T}=w_{r}+i w_{i}$ with $v_{T} \equiv$ $\equiv\left(2 k_{\mathrm{B}} T / m\right)^{1 / 2}$, and $Q_{1}(\xi) \equiv(\xi / 2) \ln [(\xi+1) /(\xi-$ $-1)]-1$ with $\xi \equiv w(1+i / \mathcal{K})$. In the analytical derivation of this dispersion equation, we used approximately $p \approx p_{T}=m v_{T}$ by the properties of the Maxwellian distribution (3) calculating the angle integrals over the momentum $\mathbf{p}$, which simplifies this equation, in contrast to the derivations in Ref. [21]. The absolute values of the sound-wave number $\beta=\omega / w_{r}$ and the scaled absorption coefficient $\gamma / \beta$ are given by

$\beta=\frac{\omega}{v_{T}} \frac{\left|w_{r}\right|}{w_{r}^{2}+w_{i}^{2}}, \quad \frac{\gamma}{\beta}=\left|\frac{w_{i}}{w_{r}}\right|$.

Thus, one obtains the wave number $\beta>0$ and the absorption coefficient $\gamma>0$ for sound waves spreading in the positive $z$-axis direction for $z>0$. Similarly, one finds the contributions of other poles [37].

Taking the asymptotic expansion of $\mathrm{D}(w, \mathcal{K})$ [Eq. (19)] in a power series of $\mathcal{K}$ within the FCR, where $\mathcal{K} \ll 1$, for the isothermal sound velocity $c$ in units of the adiabatic sound velocity $c_{0}[\mathrm{Eq} .(1)]$, one finds

$\frac{c}{c_{0}} \cong \frac{4}{\sqrt{15 \pi}}+a_{2}(\omega \tau)^{2}+O\left[(\omega \tau)^{4}\right]$,

$\frac{\gamma}{\beta_{0}} \cong \frac{(21 \pi-40) \sqrt{15 \pi}}{160} \omega \tau+O\left[(\omega \tau)^{3}\right]$,

where $a_{2} \cong 0.67 \quad\left(\beta_{0}=\omega / c_{0}\right)$. In the RCR, $\mathcal{K} \gg 1$, the asymptotic expansion of Eq. (19) in $1 / \omega \tau$ yields

$$
\begin{aligned}
\frac{c}{c_{0}} \cong\left[1-\frac{1}{(\omega \tau)^{2}}\right] \sqrt{\frac{6}{5}}+O\left[(\omega \tau)^{-4}\right], \\
\frac{\gamma}{\beta_{0}} \cong \sqrt{\frac{5}{6}} \frac{1}{\omega \tau}+O\left[(\omega \tau)^{-4}\right] .
\end{aligned}
$$

\section{Comparison with Data}

In this section, we present a comparison of the obtained theoretical results with the available data 
$[9,12]$ on the speed and absorption coefficient of APSWs. These data were originally presented in terms of the dimensionless variable $[9,10,12]$

$r=\frac{2 f_{c}}{5 \pi f} \equiv \frac{4 f_{c}}{5 \omega}$

where $f_{c}=\bar{v} / l$, which coincides with $\tau^{-1}$ given by Eq. (6). We prefer to use the quantity $\omega \tau \equiv 0.8 r^{-1}$. Thus, in contrast with the original presentation, the frequency $\omega$ in our figures is increasing from left to right along the abscissa axis.

The fundamental experimental observation consists in the following. All monoatomic gases with different values of a mass $m$, diameter $d$ (cross-section $\sigma=\pi d^{2}$ ), density $n$, and temperature $T$, i.e., different equilibrium states of the gas, have the same universal behavior of the speed of sound and the scaled absorption coefficient presented in terms of the dimensionless quantity $\omega \tau$. The data at different $m, \sigma$, $T$, and $n$ values can be shown at the same figure, but with $\tau=\tau(m, \sigma, n, T)$ calculated by Eq. (6). We should only remember that the same value of $\omega \tau$ may correspond to different $\omega$ and $\tau=\tau(m, \sigma, n, T)$ values. Our description is in complete agreement with this experimentally observed scaling. Note that, in Ref. [9], the pressure $P$ was changed from 1 atm to $10^{-2}-10^{-3}$ atm. In addition to $\mathrm{Ar}$ and Ne atoms presented in Figs. 1 and 2, the measurements were also done for $\mathrm{He}, \mathrm{Kr}$, and $\mathrm{Xe}$.

Figure 2 shows the sound velocity $c / c_{0}(a)$ and the scaled absorption coefficient $\gamma / \beta_{0}(b)$ as functions of the Knudsen parameter $\omega \tau$. The results presented by solid lines are obtained numerically by solving the dispersion equation (19). In the both limits $\omega \tau \ll 1$ and $\omega \tau \gg 1$, our numerical results converge [37] to the asymptotic results of the FCR and RCR, respectively. These limiting behaviors correspond to Eqs. (22) and (24) at leading (quadratic) orders.

As seen from Fig. 2, $a, c / c_{0}$ increases in a transition region from the FCR to the RCR, where $\omega \tau \sim$ $\sim 1$. This theoretical result is in a qualitative agreement with the data. Figure 2, a shows, however, the numerical discrepancies for the absolute values of $c / c_{0}$. Their main reason is a well-known difference between isothermal and adiabatic limits of the sound velocity [1]. The comparison with the experimental results is improved for a relative sound dispersion [17], $\left(c / c_{0}\right)(15 \pi)^{1 / 2} / 4$, i.e., for the $\omega \tau$-dependent deflec-
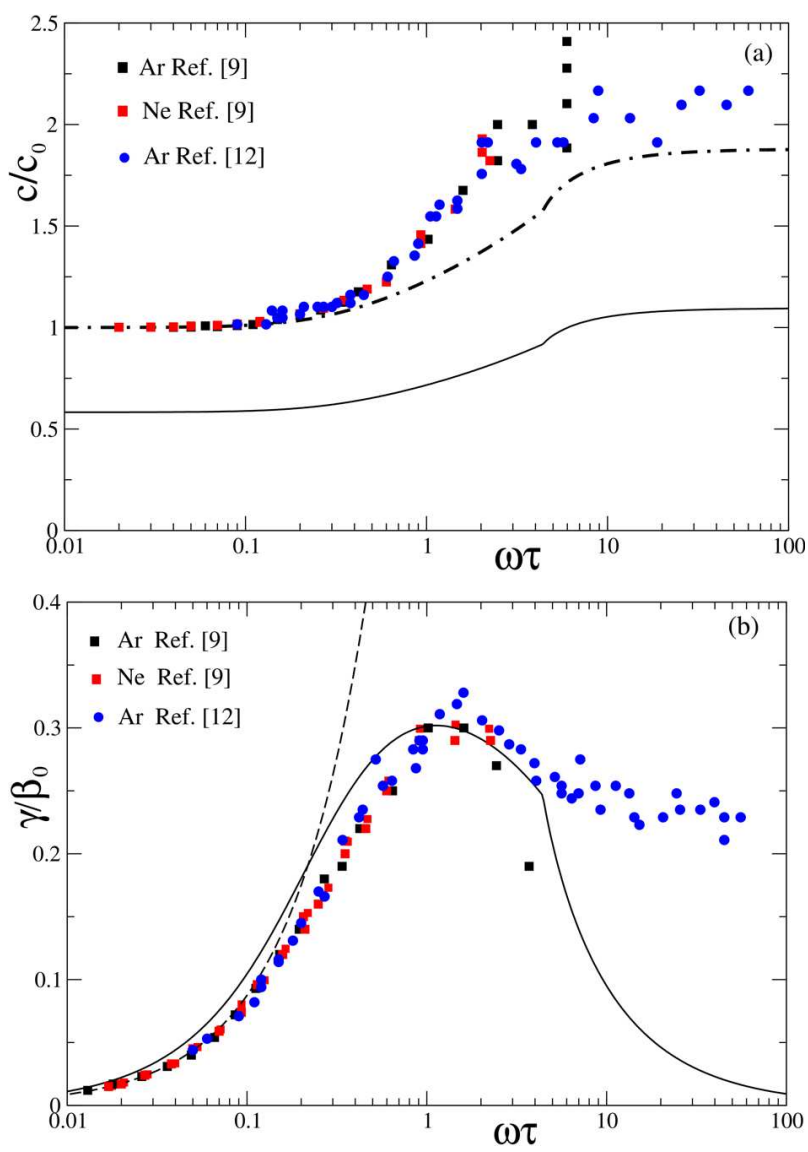

Fig. 2. Sound velocity $c(a)$ in units of $c_{0}(1)$ and the scaled absorption coefficients $\gamma / \beta_{0}(b)$ as functions of $\omega \tau$. Solid black lines show the nonperturbative solutions to the dispersion equation (19). Dash-dotted line in $(a)$ is the relative sound velocity $\left(c / c_{0}\right)(15 \pi)^{1 / 2} / 4$. Dashed line in $(b)$ presents the Stokes equation (9) calculated in the FCR

tion of the sound velocity $c$ from its FCR limit, see the dash-dotted line in Fig. 2, $a$.

Figure 2, $b$ shows the scaled absorption coefficient $\gamma / \beta_{0}$ [Eq. (20)]. The absorption coefficient $\gamma / \beta_{0}$ as a function of the Knudsen parameter demonstrates a maximum at $\omega \tau \sim 1$ in the transition from the FCR to the RCR. This behavior is also in qualitative agreement [see Fig. 2, b] with the experimental data $[9,12]$. For even larger $\omega \tau$, one finds a significant difference from the results of Ref. [12]. The "kink" point in the dependence of the scaled absorption coefficient $\gamma / \beta$ and of the sound velocity $w_{r}$ on the Knudsen parameter $\mathcal{K}$ is found numerically to the right of the maximum at $\omega \tau \approx 4.47$, where their derivatives with respect to $\omega \tau$ are sharply 
changed. This is obtained in the numerical calculations which are carefully checked within two different numerical schemes. It is worth to note that the branch point (b.p.) of the Legendre function $Q_{1}(\xi)$ of the second kind (zero of its logaritmic presentation) in the dispersion equation (19) is determined by $\xi=1$, that means $w_{\text {b.p. }}=\mathcal{K} /(\mathcal{K}+i)$ for the sound velocity. This corresponds to the scaled absorption $\gamma / \beta$ at the branch point $(\gamma / \beta)_{\text {b.p. }}=1 / \mathcal{K}$. However, we did not find analytically the Knudsen parameter $\mathcal{K}$ at the branch point. There are two length scales in the problem: the mean free path of particles in a gas, $l=v_{T} \tau$, and the sound wavelength, $\lambda=w_{r} v_{T} 2 \pi / \omega$. The "kink" corresponds to the $\omega \tau$ point, where these two different scales become approximately equal, $l \approx \lambda$. This takes place in the nonperturbative region of $\omega \tau$ values. Nevertheless, the branch point $w_{\text {b.p. }}(\mathcal{K})$ as a function of $\mathcal{K}$ coincides with the asymptotic RCR solution $w_{\mathrm{RCR}}=\mathcal{K} /(\mathcal{K}+i)$ found in Ref. [37] for large $\mathcal{K}$ and, thus, one obtains approximately $\gamma / \beta=1 / \mathcal{K}$ starting from this "kink" point. Such a presence of the "kink" point resembles a situation similar to phase transitions in statistical mechanics. An origin of the "kink" remains the open problem that deserves further studies.

Note that, in the RCR, $\omega \tau \gg 1$, one finds $\gamma^{-1} \sim l$ from Eq. (24), i.e., the propagating length of the plane sound waves is of the order of a mean-free path in a gas. The quantity $l[$ Eq. (5)] remains rather small even for dilute gases. In a gas under the normal conditions, the mean-free path is estimated as $l \sim 10^{-5} \mathrm{~cm}$. Even at much small pressures, e.g., $P \sim 10^{-3}$ atm, the propagation length $\gamma^{-1} \sim l \sim$ $\sim 10^{-2} \mathrm{~cm}$ remains, in fact, rather small at $\omega \tau \gg 1$. On the other hand, the Stokes formula (2) was obtained from the transport equation for the entropy density [1] under the assumption of a weak absorption, $\gamma \lambda /(2 \pi) \ll 1$. In the RCR, this estimate is still valid, as $\lambda \ll 2 \pi l$. Therefore, one may expect the validity of Eq. (2) in the RCR as well. This requires, however, a strong modification of the kinetic coefficients which become also dependent on the sound frequency $\omega$ (see Ref. [36]).

\section{Summary}

The kinetic approach based on the linear response theory for the BKE is developed to calculate the velocity and absorption coefficient for the plane sound waves. Our solution is based on the relaxation time approximation of the Boltzmann collision integral for the classical dilute gases.

Nonperturbative numerical solutions are found for the sound velocity and absorption coefficient as functions of the Knudsen parameter $\omega \tau$. They agree with the asymptotic expansions in both FCR and RCR approximations. Our results are in agreement with experimentally observed scaling, which means a dependence of both sound wave quantities - velocity $c / c_{0}$ and absorption coefficient $\gamma / \beta_{0}$ - on the single dimensional parameter $\omega \tau$. Qualitative changes of the sound velocity and scaled absorption coefficient in the transition region $\omega \tau \sim 1$ are observed: The sound velocity $c / c_{0}$ strongly increases, while the absorption coefficient $\gamma / \beta_{0}$ has a maximum at $\omega \tau \approx 1$. Both these theoretical results are in agreement with the data.

Our theoretical description is not complete. The presented BKE calculations should be extended to account for the thermal conductivity effects. In the RCR, the experimental values of $\gamma / \beta_{0}$ seem to be essentially larger than our estimate $\sim(\omega \tau)^{-1}(24)$ at $\omega \tau \gg 1$. This can be a signal of a presence of additional physical mechanisms for the sound-wave suppression which were not included in the present consideration.

As for the perspective, one possible application of the kinetic theory in the RCR is high-energy nucleusnucleus collisions. The intermediate stage of these collisions is often described by the hydrodynamic approach. The hydrodynamic description should be stopped at some stage (the so-called freeze-out procedure). After such a stage, the system is usually considered as one of freestreaming particles. At this postfreeze-out stage, however, the particle collisions still occur, and the final momentum spectra are influenced by these collisions. This stage is the RCR of the kinetic models. The mean free path of particles flying away becomes larger than system's size.

We thank S.N. Reznik, P. Ring, and A.I. Sanzhur for fruitful discussions. The work of A.G.M. on the project "Nuclear collective dynamics for high temperatures and neutron-proton asymmetries" was supported by the Program "Fundamental research in high energy physics and nuclear physics (international collaboration)" at the Department of Nuclear Physics and Energy of the National Academy of Sciences of Ukraine. The work of M.I.G. was supported by the 
Program of Fundamental Research of the Department of Physics and Astronomy of the National Academy of Sciences of Ukraine.

1. L.D. Landau, E.M. Lifshitz. Fluid Mechanics (Pergamon Press, 1987).

2. K.B. Tolpygo. Thermodynamics and Statistical Physics (Kiev University, 1966) (in Russian).

3. J.H. Fertziger, H.G. Kaper, Mathematical Theory of Transport Processes in Gases (North-Holand, 1972).

4. S. Chapman, T.G. Cowling, The Mathematical Theory of Non-Uniform Gases (Cambridge Univ. Press, 1990).

5. E.M. Lifshitz, L.P. Pitaevskii. Physical Kinetics (Elsevier, 1981).

6. L. D. Landau, E. M. Lifshitz. Statistical Physics (Pergamon, 1980).

7. C. Cercignani. Mathematical Methods in Kinetic Theory (Springer, 1969).

8. C. Cercignani. Theory and Application of the Boltzmann Equation (Scottish Academic Press, 1975).

9. M. Greenspan. Propagation of sound in five monatomic gases. J. Acoust. Soc. Am. 28, 644 (1956).

10. M. Greenspan. Transmission of sound waves in gases at very low pressures. In: Physical Acoustics, edited by W.P. Mason (Academic, 1965), Vol. II, Part A.

11. R. Schotter. Rarefied gas acoustics in the noble gases. Phys. Fluids 17, 1163 (1974).

12. M.E. Meyer, G. Sessler. Schallausbreitung in Gasen bei hohen Frequenzen und sehr niedrigen Drucken. Z. Phys. 149, 15 (1957).

13. C.S. Wang Chang. On the Dispersion of Sound in Helium. Report APL/JHU CM-467, UMH-3-F (University of Michigan, 1948).

14. C.S. Wang Chang, G.E. Uhlenbeck. The heat transport between two parallel plates as function of the Knudsen number. In: Studies in Statistical Mechanics. Edited by J. de Boer and G.E. Uhlenbeck (Elsevier, 1970), Vol. V, pp. $43-75$.

15. L.C. Woods, H. Troughton. Transport processes in dilute gases over the whole range of Knudsen numbers. Part 2. Ultrasonic sound waves. J. Fluid. Mech. 100, 321 (1980).

16. G. Lebon, A. Cloot. Propagation of ultrasonic sound waves in dissipative dilute gases and extended irreversible thermodynamics. Wave Motion 11, 23 (1989).

17. M.N. Kogan, Dynamics of the Dilute Gas. Kinetic Theory (Nauka, 1967) (in Russian).

18. P.L. Bhatnagar, E.P. Gross, M. Krook. A model for collision processes in gases. I. Small amplitude processes in charged and neutral one-component systems. Phys. Rev. 94, 511 (1954).

19. E.P. Gross, E.A. Jackson. Kinetic models and the linearized Boltzmann equation. Phys. Fluids 2, 432 (1959).

20. H. Heiselberg, C.J. Pethick, D.G. Revenhall. Collective behavior of stable and unstable hot Fermi systems. Ann. Phys. (N. Y.) 223, 37 (1993).
21. M. Takamoto, S. Inutsuka. The relativistic kinetic dispersion relation: Comparison of the relativistic BhatnagarGross-Krook model and Grad's 14-moment expansion. Prog. Theor. Phys. 123, 903 (2010).

22. A.G. Magner, D.V. Gorpinchenko, J. Bartel. A semiclassical collective response of heated, asymmetric, and rotating nuclei. Phys. At. Nucl. 77, 1229 (2014).

23. L. Sirovich, J.K. Thurber. Propagation of forced sound waves in rarefied gasdynamics. J. Acoust. Soc. Am. 37, 329 (1965).

24. J.K. Buckner, J.H. Ferziger. Linearized boundary value problem for a gas and sound propagation. Phys. Fluids 9, 2315 (1966).

25. J.R. Thomas Jr., C.E. Siewert. Sound-wave propagation in a rarefied gas. Transp. Theory Statist. Phys. 8, 219 (1979).

26. S.K. Loyalka, T.C. Cheng. Sound-wave propagation in a rarefied gas. Phys. Fluids 22, 830 (1979).

27. T.C. Cheng, S.K. Loyalka. Sound wave propagation in a rarefied gas-II: Gross-Jackson model. Prog. Nucl. Energy 8, 263 (1981)

28. R.D.M. Garcia, C.E. Siewert. The linearized Boltzmann equation: Sound-wave propagation in a rarefied gas. Z. angew. Math. Phys. 57, 94 (2005).

29. F. Sharipov, D. Kalempa. Numerical modeling of the sound propagation through a rarefied gas in a semi-infinite space on the basis of linearized kinetic equation. J. Acoust. Soc. Am. 124, 1993 (2008).

30. I.L. Bekharevich, I.M. Khalatnikov. Theory of Kapitsa heat jump on a solid boundary. Sov. J. Exp. Theor. Phys. 12, 1187 (1961).

31. Yu.B. Ivanov. Fermi-liquid surface vibrations. Nucl. Phys. A 365, 301 (1981).

32. A.G. Magner. Boundary conditions for semiclassical distribution function. Sov. J. Nucl. Phys. 45, 235 (1987).

33. N.G. Hadjiconstantinou, A.L. Garcia. Molecular simulations of sound wave propagation in simple gases. Phys. of Fluids 13, 1040 (2001).

34. V.M. Strutinsky, S.M. Vydrug-Vlasenko, A.G. Magner. Macroscopic resonances in planar geometry. Z. Phys. A 327, 267 (1987).

35. V.M. Kolomietz, A.G. Magner, V.M. Strutinsky, S.M. Vydrug-Vlasenko. Monopole modes in a finite Fermi system with diffuse reflection boundary conditions. Nucl. Phys. A 571, 117 (1994).

36. A.G. Magner, M.I. Gorenstein, U.V. Grygoriev. Viscosity of a classical gas: The rare-collision versus the frequentcollision regime. Phys. Rev. E 95 (2017) 052113.

37. A.G. Magner, M.I. Gorenstein, U.V. Grygoriev. Ultrasonic waves in classical gases. Phys. Rev. E 96, 062142 (2017).

38. D. Forster, Hydrodynamic Fluctuations, Broken Symmetry and Correlations Functions (Benjamin, 1975).

39. D. Zubarev, V. Morozov, G. Röpke. Statistical Mechanics of Non-Equilibrium Processes (Fizmatlit, 2002), Vol. II (in Russian).

40. V.P. Silin. Introduction to the Kinetic Theory of Gases (Nauka, 1971) (in Russian).

Received 23.09.19 
А.Г. Магнер, М.І. Горенштейн, У.В. Григор'єв

ШВИДКІСТЬ І КОЕФІЦІЕНТ

ПОГЛИНАННЯ ЗВУКОВИХ ХВИЛЬ

В КЛАСИЧНИХ ГАЗАХ

Р е $з$ ю м е

Знайдено швидкість і коефіцієнт поглинання плоских звукових хвиль в класичних газах шляхом розв'язку кінетичного рівняння Больцмана. Це зроблено в рамках теорії лінійного відгуку одночастинкової функції розподілу на пе- ріодичне зовнішнє поле. Непертурбативне рівняння дисперсії отримано в наближенні часу релаксації і розв'язано чисельно. Отримані теоретичні результати показують універсальну залежність швидкості звуку і коефіцієнта поглинання від параметра $\omega \tau$, де $\omega$ - частота звуку, а $\tau^{-1}-$ частота зіткнень частинок. В області $\omega \tau \sim 1$ відбувається зміна режиму від частих зіткнень до поодиноких, швидкість звуку різко збільшується, і коефіцієнт поглинання має максимум - обидва теоретичні ефекти відповідають експерименту. 